\title{
Survey on ELISA Based on anti Influenza A NS1 Antibodies to Differentiate the Infected and Vaccinated Poultries
}

\author{
Forough Talazadeh ${ }^{1, *}$, Mansoor Mayahi $^{1}$, Masoudreza Seifi $^{2}$, Mehdi Pourmehdi $^{3}$ \\ ${ }_{1}^{1}$ Department of Clinical Sciences, Faculty of Veterinary Medicine, Shahid Chamran University of Ahvaz, Ahvaz, IR Iran \\ 2 Department of Pathobiology, Faculty of Veterinary Medicine, Shahid Chamran University of Ahvaz, Ahvaz, IR Iran \\ 3 Department of Food Hygiene and Quality control, Faculty of Veterinary Medicine, Shahid Chamran University of Ahvaz, Ahvaz, IR Iran \\ ${ }^{*}$ Corresponding author: Forough Talazadeh, Department of Clinical Sciences, Faculty of Veterinary Medicine, Shahid Chamran University of Ahvaz, Ahvaz, IR Iran. Tel: +98-6113330073 \\ Fax:+98-6113360807, E-mail: ftalazade@gmail.com.
}

Received: July 02, 2012; Revised: August 08, 2012; Accepted: August 13, 2012

\begin{abstract}
Background: The Vaccination programs to control avian influenza (AI) infection in poultry, have limitations due to the difficulty in differentiating the vaccinated and naturally infected birds AI vaccination would bring greater global acceptance if a reliable test, which could clearly distinguish the naturally infected and vaccinated-only animals (DIVA), was available. Since the nonstructural protein (NS1) is expressed in influenza infected cells, and it is not presented as a virion, it could be a proper candidate for DIVA differential diagnostic test. Objectives:Vaccination programs for the control of avian influenza (AI) in poultry have limitations due to the problem of differentiating between vaccinated and virus-infected birds. The use of AI vaccination in poultry would have greater worldwide acceptance if a reliable test were available that clearly discriminated between naturally infected and vaccinated-only animals (DIVA). Because the nonstructural protein (NS1) is expressed in influenza virus-infected cells, and it is not packaged in the virion, it is an attractive candidate for a DIVA differential diagnostic test.

Materials and Methods: A total of 300 day-old broiler chicks (Ross 308) divided into three equal groups (1 to 3).The chicks in group 1 were immunized with killed AIV H9N2. The chicks in group 2 were infected with AI virus subtype H9N2. The chicks in group 3 were kept as controls and did not receive any vaccined or lived virus. Chicks sera were collected at day 42 usingrNS1-ELISA and Commercial ELISA kit. Results: Designed ELISA test for detection of antibody against influenza NS1 could experimentally distinguish the chicks infected with AIV and chicks immunized with killed influenza viruswith93.3\% sensitivity and $100 \%$ specificity. 3 weeks after infection or vaccination, sera from all two treated groups were positively tested using commercial ELISA kit (IDEXX). In contrast, by NS1-ELISA, only infected groups sera were tested and the result was positive, and all sera samples from the vaccinated group were NS1-antibody titer were evaluated and the result were negative.
\end{abstract}

Conclusions:: It was concluded that antibodies against AIV NS1 protein was only detected in the sera of chickens experimentally infected with AIV, not in the sera of chickens immunized with inactivated vaccine.

Keywords: Avian Influenza Virus (AIV); Nonstructural Protein (NS1); Antibody; ELISA

\section{Background}

Influenza viruses are segmented, negative-strand RNA viruses belonging to the family of Orthomyxoviridae and they are divided in three major types, A, B, and C (1). All three types of influenza are found in humans, but only the Influenza type A viruses are found in birds and are called "avian influenza (AI) viruses". Type A influenza viruses are divided into subtypes based on the antigenic properties of the major surface glycoproteins: the hemagglutinin (HA) and neuraminidase (NA) proteins. In total, 16 HA sub types (H1-H16) and nine neuraminidase subtypes (N1-N9) are described at present (1). H9N2 virus shares similar receptor binding epitopes with human influenza viruses, witha broader host range, and human infections have been reported. In addition, the latency of H9N2 AIV infection in chickens caused this infection to be easily overlooked and increases the chance of human infection.

Although AI vaccines have been effective in providing protection against clinical signs and death among poultry (2-4), they are not commonly used as part of control and eradication programmes of LPAI or HPAI virus infections (3). For a number of reasons, including the concerns over poultry and poultry products trade embargoes, quarantine and elimination of infected flocks (i.e. stamping out or controlled marketing) are often desirable measurements to control AI. An additional limitation is that traditional AI virus vaccines can interfere with serologic surveillance; hence, these vaccines induce antibodies that are indistinguishable from the antibodies produce in live virus infected birds, as determined by commercial enzyme-linked immunosorbent assays (ELI-

Implication for health policy/practice/research/medical education:

The study provides guidelines for researchers to develop the NS1-ELISA as a potentially valuable diagnostic technique to AIabolition and control programs and this specific ELISA for anti-NS1 antibodies may have diagnostic value in the poultry industries.

Copyright (C) 2013, Ahvaz Jundishapur University of Medical Sciences; Licensee Kowsar Ltd. This is an Open Access article distributed under the terms of the Creative Commons Attribution License (http://creativecommons.org/licenses/by/3.0), which permits unrestricted use, distribution, and reproduction in any medium, provided the original work is properly cited. 
SAs), and hemagglutination inhibition assays (HAI) $(5,6)$. Therefore, the efforts have been focused on developing new serologic tools to allow differentiation of infected from vaccinated animals, which is commonly known as the DIVA strategy.

For international commerce of vaccinated birds, the DIVA strategy needs to not only differentiate infected and vaccinated birds, but also to identify vaccinated AIV infected birds. Several different DIVA strategies have been proposed (7), some of which require appropriate vaccines and specific companion serologic discriminatory tests (8-12). This sort of strategy is important to provide a guarantee to trading partners to safely trade influenza free poultry products. The DIVA strategy has been accepted by some European countries in as an approach to monitor and provide assurance to the infection-free status of vaccinated poultry during an LPAI outbreak (7, 13). One potential target for the DIVA strategy for avian influenza virus is the nonstructural (NS1) protein. The NS1 protein is encoded by the smallest gene segment of influenza virus, segment 8 , and which has a critical role in suppression of interferon induction in the host cells that increases the virus replication efficiency(14). The NS1 protein is a conserved protein amongst type A influenza viruses, but it compromises of two major subtypes, A and B, which differ by about 35\% of nucleotides (15).

The NS1 protein is produced during influenza virus replication in infected cells $(12,16)$, but it is not packaged into the infectious viral particle, being a true nonstructural protein. Because most AIV vaccines are mostly chemically inactivated, vaccinated animals theoretically should not express humoral response to the NS1 protein, but infected poultries secret anti NS1 antibodies at high levels. This NS1 DIVA approach was first proposed for equine influenza viruses management, and purified NS1 protein was used to detect antibodies against NS1 in serum samples from ponies and horses experimentally infected with influenza virus $(16,17)$. Serologic studies on chickens and turkeys using an ELISA with AIV NS1 protein produced in Escherichia coli as antigen indicated that infected birds showed higher levels of NS1 antibody as compared to vaccinated birds $(12,18)$.

\section{Materials and Methods}

\subsection{Chickens}

A total of 300 day-old broiler chicks (Ross 308 ) were provided. All chickens were housed in 3 different places and breed under high standard condition.

\subsection{Virus}

The AI viruses used in this study (A/Chicken/Iran/AH1/06), subtype H9N2 were isolated from a broiler chicken flock in Ahvaz, with respiratory signs and mortality. These viruses were initially isolated in 10-day-old embryonated chicken eggs (ECE). They were sub typed by standard hemagglutination-inhibition and neuraminidase-inhibition tests with polyclonal chicken antisera. The viruses were passaged in ECE and the allantoic fluids were harvested as stock viruses for further analysis.

\subsection{Vaccines}

AIV vaccine consisting of inactivated commercial AI vaccines, subtypes H9N2.

\subsection{Experimental Design}

The chickens divided into three equal groups (1 to 3 ). The chicks in groups 1 were immunized subcutaneously with AIV H9N2inactivated virus in neck. The chicks in groups 2 were intra nasally infected with infectious $0.2 \mathrm{ml}$ allantoic fluid. The chicks in groups 3 were kept as control group and did not receive vaccine or live virus. Chicks sera were collected at day 42 used in ELISA test design.

\subsection{Production and Purification of Recombinant NS1}

Coding region of NS1 gene of avian influenza virus isolate (A/Chicken/Iran/AH-1/06), serotype H9N2 from a broiler chicken flock in Ahvaz, with respiratory symptomsand mortality, was amplified using RT-PCR and was expressed in BL strain of E.coli after cloning in pMAL-C2X vector. For this purpose, several nucleotide sequence of the NS1 coding region (from nucleotide 27 to 680) of avian influenza virus subtype H9N2 were extracted from the Gene bank and the required primers were designed. The NS1 gene was subsequently amplified by RT - PCR. The vector, pMAL-C2X and the amplified NS1 gene, both were digested by Eco RI and PstI and ligated by T4 ligase. The recombinant construct was transformed into BL strain of E.coli and finally the purified recombinant vector and used for expression of NS1 protein. Viral ribonucleic acid (RNA) was extracted. A pair of primers was designed according to the sequence of the influenza virus nonstructural protein (NS1) and a 654 base pair (bp) gene segment was amplified by reverse transcription-polymerase chain reaction (RT-PCR) with the template of influenza virus (H9N2) isolated.

NS1-specific primers forward: $5^{-}$-GCCGGAATTCATGGATTCCAACACTGTG-3-

NS1-specific primers reverse: $5^{\prime}$-GCCCCTGCAGCTACTTTGGAGAGAGTGT- $3^{-}$

The amplified DNA fragment sequences were searched in GenBank by using the basic local alignment search tool (Bioedit).to express the protein, E.coli clones of pMAL-C2X with the NS1 inserts were grown in LB broth, supplemented with glucose and $100 \mu \mathrm{g}$ of ampicillin per $\mathrm{mL}$ at $37^{\circ} \mathrm{C}$ in a shaking incubator at $250 \mathrm{rpm}$. IsopropylD-thiogalactopyranoside ( $1 \mathrm{mM}$ ) was added to the culture (LB broth, supplemented with glucose and $100 \mu \mathrm{g}$ of ampicillin) with an absorbance of $0.5 \mathrm{aOD} 600 \mathrm{~nm}$ and the 
tubes were incubated for an additional $3 \mathrm{~h}$. The culture fluid was centrifuged at $6000 \mathrm{~g}$ for $10 \mathrm{~min}$ at $4^{\circ} \mathrm{C}$ in an SLA3000 rotor in a Sorvall RC 5B plus centrifuge. The supernatants were discarded, and the cells were re-suspended in buffer containing $20 \mathrm{mM}$ Tris- $\mathrm{HCl}$ (pH 7.4), $200 \mathrm{mM}$ $\mathrm{NaCl}$, and $1 \mathrm{mM}$ EDTA. The cell pellet was thawed and lysed by sonication on ice at $15 \mathrm{~W}$ in $20 \mathrm{~s}$ pulses. The lysate was centrifuged in a Sorvall SLA-1500 rotor at $18000 \mathrm{~g}$ for 30 $\min$ at $4^{\circ} \mathrm{C}$

\subsection{Chromatography}

The lysate supernatant was loaded on to the amylose affinity resin in a Kontes column (10 by $1.75 \mathrm{~cm})$. The column was washed with 2 volumes of column buffer, and the MSB-NS1 fusion protein was eluted with column buffer plus $10 \mathrm{mM}$ maltose. The fractions containing purified protein were collected, aliquoted, and stored at -80 ${ }^{\circ} \mathrm{C}$.

\subsection{SDS-PAGE and Western Blotting Assay}

The purified protein was analyzed by 10\% SDS-PAGE, identified either by dying directly with Coomassie brilliant R250 or transferred to nitrocellulose membrane for Western blot detection. The membrane was blocked over night at $4{ }^{\circ} \mathrm{C}$ in PBS- Tween containing 5\% skim milk Subsequently, the primary serum antibody of chickens infected with H9N2 AIVincubated for $1 \mathrm{~h}$ at room temperature.

\subsection{ELISA for NS1 Detection}

Serum samples were diluted in titer tubes 1 day before the test. The diluent serum consisted of 5\% (wt/vol) nonfat skim milk containing $2 \%$ normal E.coli cell lysate in PBSTween. The E.coli cell lysate was removed from the serum diluent used onNS1 peptide-coated ELISA plates. E. coli cell lysate were added to the diluent serum to eliminate nonspecific reactions. The optimum dilution concentration of theNS1 protein for the ELISA test was obtained by checkerboard titration on a 96-well ELISA plate against known positive and negative samples. The highest antigen dilution that saturated the plate and gave maximum contrast in terms of optical density (OD) between known positive and negative sera considered as the optimum dilution concentration. The optimum considered dilution was the highest antigen dilution that saturated the plate and gave maximum contrast in terms of optical density (OD) between known positive and negative sera.

The experimental serum samples with the dilution of 1:400were determined to produce an optimal level of sensitivity for detection of positive serum samples with low background in negative samples. Indirect ELISA was performed using the following procedure. Ninety-six well ELISA plates were coated with $0.299 \mu \mathrm{g} /$ well purified NS1 protein diluted in $100 \mu \mathrm{L}$ of coating buffer ( $0.1 \mathrm{M}$ carbonate/bicarbonate buffer, $\mathrm{pH}$ 9.6) per well. After an over- night incubation, the plates were three times washed by PBS with 5\% Tween, and the wells were blocked with $300 \mu \mathrm{L}$ blocking buffer (PBS with 5\% skim milk) and incubated for $2 \mathrm{~h}$ at $37^{\circ} \mathrm{C}$. The plates were washed four times by PBS with $5 \%$ Tween. Each test sample was 1:400 diluted in Sample Dilution Buffer (The serum diluent for the recombinant NS1 protein, ELISA consisted of 5\% (wt/vol) nonfat skim milk containing $2 \%$ normal E.coli cell lysate in PBS-Tween) and $100 \mu \mathrm{L}$ were added to the ELISA plate and incubated for $1 \mathrm{~h}$ at $37^{\circ} \mathrm{C}$.

The plates were washed four times by PBS with $5 \%$ Tween .peroxidase-conjugated to the goat anti-chicken antibodies 1:10000 diluted in conjugate diluent Buffer (0.5\% (wt) vol) nonfat skim milk containing $1 \%$ rabbit serum in PBSTween) and $100 \mu \mathrm{L}$ were added to each well and incubated for $1 \mathrm{~h}$ at $37^{\circ} \mathrm{C}$. The plates were washed four times by PBS with5\% Tween. After extensive washing, 3,3,5,5-tetramethyl benzidine (TMB; Sigma)was added as substrate. the reaction was stopped with $1 \mathrm{M} \mathrm{HCl}$ at room temperature, for $15 \mathrm{~min}$ in a dark room.. Absorbency measurement was performed at $450 \mathrm{~nm}$ using a microplate reader (Milton Roy, England). The cut-off value was calculated from the result of 96 sera of control chickens using the optical density formula (OD) (2 X mean OD). A serum sample was considered positive when its OD was greater than the cutoff.

\subsection{Commercial ELISA}

We performed Commercial ELISA assay in u-bottomed 96-well microtiter plates with commercial ELISA kit(IDEXX comanufactured in United States.).

\subsection{Hemagglutination Inhibition Assay (HAI)}

Beta procedure of hemagglutination inhibition (HAI) assays was performed in U-bottomed 96-well microtiter plates with $5 \%$ chicken erythrocytes (19).

\subsection{Sensitivity and Specificity of NS1-ELISA}

The 180 serum samples of immunized chickens and infected chickens were all positive by HI assay. With rNS1ELISA, 84 out of 90 samples from infected chickens were positive and six samples were negative, and all $90 \mathrm{immu-}$ nized samples were negative .Using HI assay as the orientation method, the sensitivity of the rNS1-ELISA was 93.3\% (84 from 90) and its specificity was $100 \%$ (90 from 90).

\section{Results}

\subsection{Preparation of Recombinant NS1}

The NS1 gene was cloned into a prokaryotic vector PMAL$\mathrm{c} 2 \mathrm{X}$ and expressed in E. coli as the production of inclusion body. The expression product was about $66.4 \mathrm{kDa}$ using SDS-PAGE (Figure 1). The protein was purified from inclusion bodies and was subjected to Western blotting anal- 
ysis using chicken H9N2 AIV antiserum and goat-antichicken secondary antibody. A single 66.4 kDa band was detected in the lysate of E. coli containing pMAL-c2X-NS1 (Figure 2, lane 2), while there was no detectable band in the lysate of E. coli containing the vector plasmid (Figure 2, lane1). Fractions 1-10 harvested from chromatography used as antigen for ELISA-NS1 test (Figure 3, lane 1-10).

Figure 1. SDS-PAGE of Recombinant NS1 Protein Stained With Coomassie Brilliant Blue R250

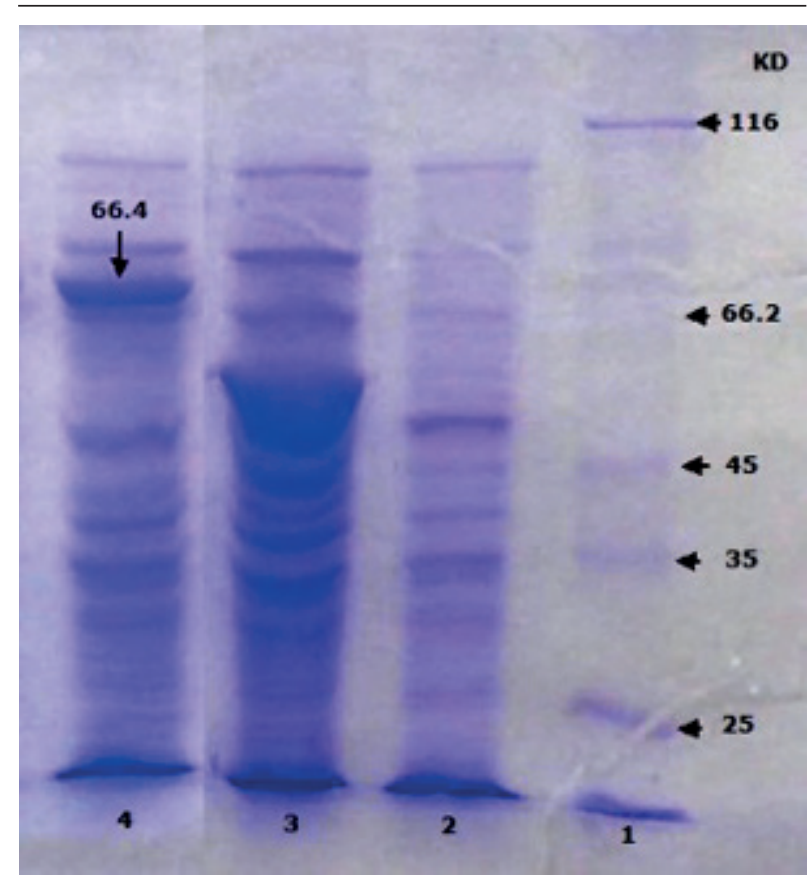

Lane 1, protein molecular weight markers; lane 2, pMAL-c2X vector before inducedbyIPTG; lane 3, pMAL-c2X vector after induced by IPTG. lane 4 PMAL- c2X-NS1 expressed in E. coli.

\subsection{Evaluation of NS1-Based Solid-Phase NS1-ELISA}

The optimal conditions for the ELISA were first established by checker board matrix titration. The amount of NS1 protein for coating ELISA plates were determined to be $0.299 \mu \mathrm{g} /$ well. The serum dilution was 1:400 that best classifies positive and negative sera with minimized false-positive or negative results. All serum samples with rNS1-ELISA were evaluated. In order to determine the best cut-off point, all sera of the control group was tested at day 42. Kolmogorov Smirnov test showed that the optical density distribution in rNS1-ELISA is normal ( $P>0.05)$ (Figure 4). One of the sera with an OD similar to the mean OD of sera was as a negative serum in the experiments and each serum that have at least twice selective Negative sera. OD were considered as positive. The cut-off rate was
0.184 while examining 96 negative sera.

Figure 2. Western Blotting of Recombinant NS1 Protein by AI-Positive Sera

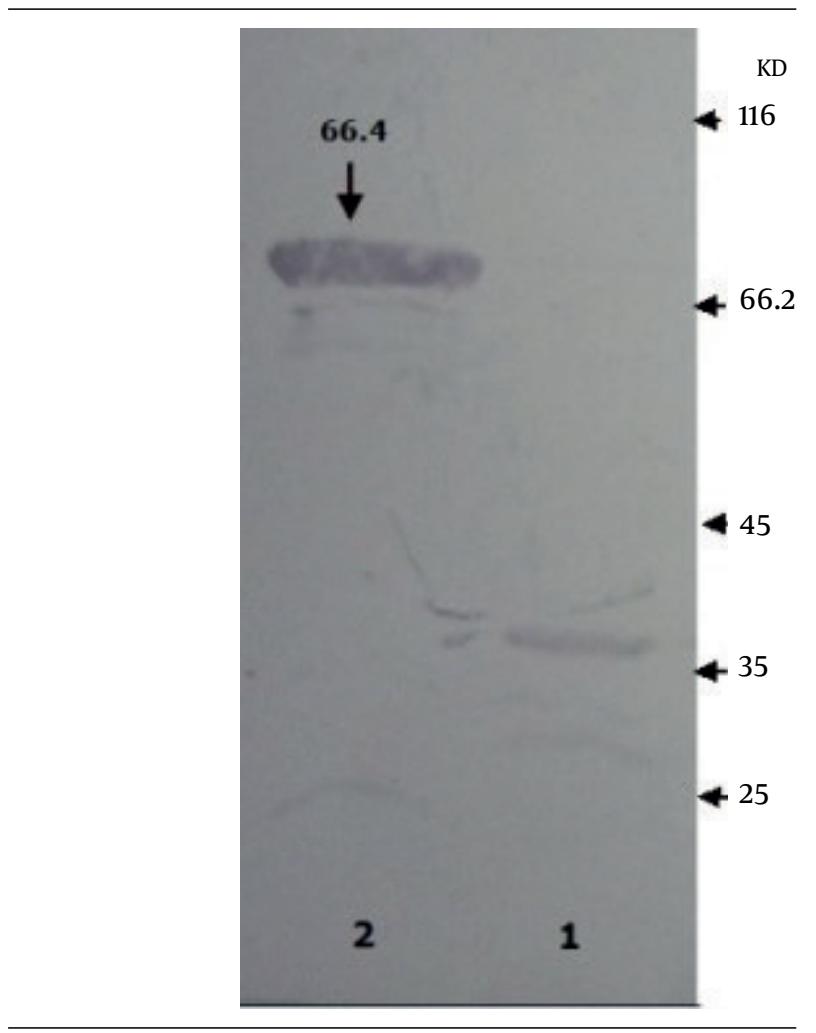

Lane 1, control of pMAL-c2X vector; lane 2, amplified protein of pMAL-c2XNS1 induced by IPTG.

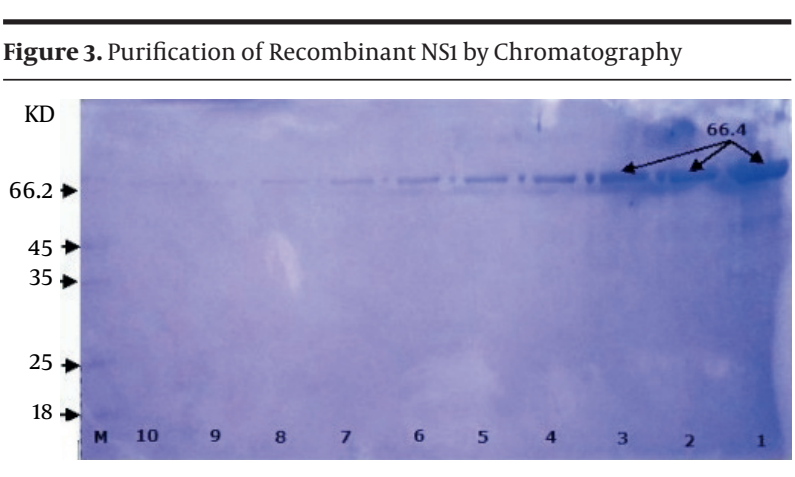

Lane M, protein molecular weight marker; lane 1- 10, purified NS1 protein in fractions

To increase specificity of the assay and reduce false positive results, which could be caused by antibodies to E. coli components, $2 \%$ E. coli lysate was added to the sample dilution buffer to absorb nonspecific antibodies against $E$. 
Talazadeh F et al.

coli (Figure 4).

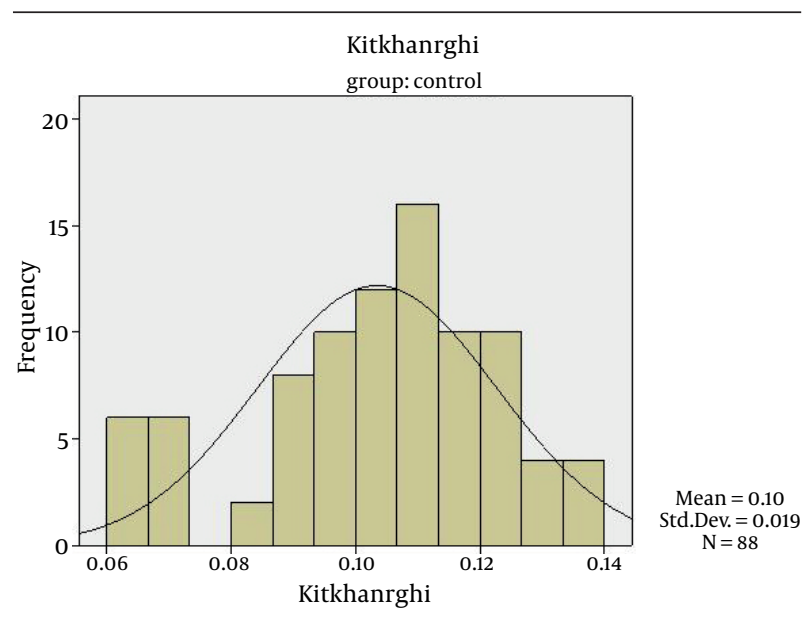

Figure 4. Kolmogorov Smirnov Test Showed That the Optical Density Distribution in rNS1-ELISA Is Designed to Be Normal

The obtained results of commercial ELISA kit (IDEXX manufactured in United States) and NS1-ELISA detecting experimental chicken sera 100 broiler chicks (Ross 308) were intramuscularly immunized (day 21) with inactivated viruses.100 broiler chicks were infected (day 21) by intranasal inoculation with $\mathrm{H} 9 \mathrm{~N} 2$ virus. The chicks collected Sera were assayed with commercial ELISA kit (IDEXX) and rNS1-ELISA. 3 wk after infection or immunization, sera from all two treatment groups tested positive with commercial ELISA kit (IDEXX). In contrast, using NS1ELISA, only sera from infected groups were positive, and all sera samples from the immunized group were NS1antibody negative. Sera from all control chickens were negative in three assessment (Tables 1 - 4).

Table 1. rNS1-ELISA Results of the Control and Treated Group Serum $^{\mathrm{a}}$

\begin{tabular}{llll}
\hline rNS1-ELISA & \multicolumn{2}{c}{ Status } & Total \\
\cline { 2 - 3 } & Treated & Control & \\
\hline Positive & 84 & 0 & 84 \\
Negative & 6 & 88 & 94 \\
Total & 90 & 88 & 178 \\
\hline
\end{tabular}

${ }^{a}$ Relative sensitivity= $(84 / 90) \times 10093.3 \%$; Relative specificity $=(88 / 88) \times 100=$ $100 \%$; Accuracy $=(84+88) / 178 \times 100=96.6 \%$; Kappa statistic $=0.933$; Positive predictive value $=(84 / 84) \times 100=100 \%$; Negative predictive value $=(88 / 94)$ $\times 100=93.6 \%$;Mc Nemar test $(P>0.05)$

Table 2. rNS1-ELISA Method Results of the Serum of Control and Immunized Group

\begin{tabular}{llll}
\hline \multirow{2}{*}{ rNS1-ELISA } & \multicolumn{2}{c}{ Status } & Total \\
\cline { 2 - 3 } & Immunized & Control & \\
\hline Positive & 0 & 0 & 0 \\
Negative & 90 & 88 & 178
\end{tabular}

$\begin{array}{llll}\text { Total } & 90 & 88 & 178\end{array}$

Table 3. Results of the Serum of Control and Treated Group in IDEXX ${ }^{\mathrm{a}}$

\begin{tabular}{llll}
\hline \multirow{2}{*}{ IDEXX } & \multicolumn{2}{c}{ Status } & Total \\
\cline { 2 - 3 } & Treated & Control & \\
\hline Positive & 90 & 0 & 90 \\
Negative & 0 & 88 & 88 \\
Total & 90 & 88 & 178 \\
\hline
\end{tabular}

${ }^{a}$ Relative sensitivity $=(90 / 90) \times 100=100 \%$; Relative specificity $=(88 / 88) \times 100=100 \%$; Accuracy $=(90+88) / 178 \times 100=100 \%$; Kappa statistic $=1$, Positive predictive value $=(90 / 90) \times 100=100 \%$; Negative predictive value $=(88 / 88) \times 100=100 \%$; Mc Nemar test $(\mathrm{P}>0.05)$

Table 4. Results of the Serum of Control and Immunized Group in IDEXX

\begin{tabular}{llll}
\hline \multirow{2}{*}{ IDEXX } & \multicolumn{2}{c}{ Status } & \multirow{2}{*}{ Total } \\
\cline { 2 - 3 } & Immunized & Control & \\
\hline Positive & 90 & 0 & 90 \\
Negative & 0 & 88 & 88 \\
Total & 90 & 88 & 178
\end{tabular}

\section{Discussion}

Since low pathogenic avian influenza (LPAI) virus infections are often asymptomatic in chickens and turkeys, the diagnosis requires serological monitoring. Most poultry diagnostic laboratories prefer to use the serologic AGP test because of its simplicity and broad specificity to detect type A influenza virus infections among poultry. AGP antibodies recognize conserved nucleoprotein (NP) and matrix (M1) influenza virus proteins presented in the sera of poultry exposed to AI virus and therefore can detect multiple subtypes of influenza A virus (20). Other serological tests for AI including ELISA, which also measures the antibody responses to conserved internal proteins, and the subtype-specific HI assay (21). However, these standard serologic tests for AI virus exposure do not differentiate immunized and infected poultry when traditional vaccines are used (5).

An improved serological assay for AI virus infections allows the identification of infected poultry with AI virus, while it may correctly exclude animals that have been immunized with any subtype. In the mentioned diagnostic test, a conserved nonstructural protein that is not associated to virions and expressed in large amounts in influenza virus-infected cells, were used (22). Following dilution of immune sera, the NS1 ELISA was an effective tool to screen the poultry sera for detection of AI virus infection. Although the NS1 protein is a weak antigen in comparison to other influenza virus proteins, but is remarkably conserved in type A influenza viruses (15, 23-25). Previous studies demonstrated that the NS1 protein could be de- 
tected in the sera of horses experimentally infected with the H3 subtype of influenza virus but not in the immune sera of animals immunized with inactivated viruses (16, 17).

The inactivated whole-virus equine vaccine, used in the fore mentioned studies was partially purified, thus removing residual NS1 protein that would be presented in the infectious medium. This allows the clear distinction between $\mathrm{H} 3$-vaccinated and $\mathrm{H} 3$-infected horses by using the NS1 protein as a differential marker $(16,17)$. BirchMachin et al. and Ozaki et al. described an ELISA application that is capable to differentiate vaccine-induced AIV antibodies from infected-induced antibodies. Antibodies against AIV NS1 protein are considered as the most useful marker of virus replication. The majority of collected sera from infected chickens, were positive, in contrast all the sera from vaccinated chickens were negative tested by NS1-ELISA and the sera from control chickens without previous exposure to AIV antigens by vaccination or infection were also negative.

The HI assay has been used as a conventional method of diagnosis to detect antibodies against influenza virus. However, the HI assay does not differentiate the antibodies induced by infection and the vaccinated ones. Theoretically, chickens immunized with inactivated virus have produced antibodies against primarily structural proteins of virus, while chickens infected with LPAI virus produced antibodies against nonstructural proteins of AIV. Thus, methods detecting antibodies against nonstructural proteins of AIV shall serve as a mean of differentiation. In the present study, we demonstrated that, by ELISA using prokaryotic (E.coli) recombinant NS1 as the antigen, anti-NS1 antibodies were only detectable in the sera of experimentally infected chickens but nothing were detected in the sera of vaccinated chickens. This observation was also reported by Tumpey et al. (12).

The reported results of Tumpey et al. indicated that the use of a simple and specific ELISA for the serodiagnosis of infection with multiple LPAI virus subtypes to distinguish between infected poultry and vaccinated poultry. In this study animals inoculated with inactivated gradient-purified vaccines had no seroconversion to positivity of antibodies against NS1 protein, and animals vaccinated with commercial vaccines had shown a low, but detectable, levels of NS1 antibodies (12). The use of a second ELISA with diluted sera identified as a diagnostic test that results in sero-positive for the antibodies against NS1 protein in infected birds (12). For the phase-field application of this study, serum samples were collected from vaccinated and infected poultry, diluted, and screened for anti-NS1 antibodies (12).

It has been observed that, the Field sera from poultry that received commercial AI killed viruses possesses antibodies against AI virus, as measured by the standard agar gel precipitin (AGP) test, but they were negative using the NS1 ELISA (12). Conversely, diluted field sera from AI-infected poultry were positive for both AGP and NS1 antibodies. These results demonstrated the potential benefit of a simple, specific ELISA for anti-NS1 antibodies with a diagnostic value in poultry industries (12).In a recent study, this observation was also reported by Zhao et al. (18). In study of Zhao et al. NS1-ELISA test was used to detect antibodies against NS1 in serum samples in comparison to $\mathrm{HI}$ assay. To evaluate the specificity and sensitivity of the NS1-ELISA, 99 serum samples were collected from experimentally manipulated chickens, including 72 samples from chickens vaccinated with inactivated vaccine and 27 samples from chickens experimentally infected with live H9N2 AIV via the subcutaneous or intramuscular injection (18).The 99 serum samples are all positive measured by HI assay. Using NS1-ELISA, all the 27 samples from infected chickens were positive, and among the 72 vaccinated samples, 68 tested negative and the rest, four samples, were weakly positive. Using HI assay as the reference method, the sensitivity of the NS1-ELISA was 100\% (27 from 27) and its specificity was 94.4\% (68 from 72) (18).

In study conducted by Avellaneda et al., an antibody response against NS1 was detected 3 weeks after infection, but the antibody levels were decreasing rapidly after 5 weeks. In vaccinated birds with inactivated oil-emulsion vaccines, antibodies against NS1 were not detected before virulent challenge, and only a small percentage of birds seroconverted after homologous LPAI virus challenge. due to the variability of seroconversion and the duration of the antibody response in chickens, the NS1 protein DIVA strategy did not perform as good as expected (26).

In a study performed by Soleimani et al.(27) the open reading frame of NS1 gene of a H9N2 subtype of AI virus was amplified by polymerase chain reaction. After extraction and agarose gel purification of NS1 gene, it inserted into two different pGEX-4T-1 and pMAL-c2X plasmids and transferred in DH5 $\alpha$ strain of E. coli by using electroporation procedure. The E.coli colonies possessing recombinant NS1 gene were screened using PCR, restriction mapping and sequencing analysis. The expressed rNS1 protein was purified using affinity chromatography based on MBP (pMAL-c2X) and GST (pGEX-4T-1). The MBP-NS1 and GST- NS1 proteins on SDS-PAGE showed bands with molecular weight of 68 and $52 \mathrm{kDa}$ respectively. Western blotting analysis with MBP-NS1 protein, showed positive reaction using antisera obtained from chickens infected with a H9N2 subtype strain. But, the western blot results of the sera collected from H9N2 immunized chickens were negative in western blotting. These findings indicated that the MBP-rNS1 protein with $26 \mathrm{kDa}$ weight expressed by PMAL-c2X plasmid can be used in a DIVA to differentiate the $\mathrm{AI}$ infected and vaccinated chickens.

In present study the results of rNS1-ELISA showed specific antibody against NS1 only detected in the sera of chicks experimentally infected with AIV. Designed ELISA test for detection of antibody against influenza NS1 could distinguish the chicks experimentally infected with AIV and chicks vaccinated with killed influenza virus with 93.3\% sensitivity and 100\%specificity. At third week after 
infection or vaccination, sera from all two treated groups were positive assayed with commercial ELISA kit (IDEXX). In contrast, by NS1-ELISA, only sera from infected groups tested positive, and all sera samples from the vaccinated group were NS1-antibody negative. Sera from all control chickens were negative in two assays.

This NS1-ELISA is useful for serological diagnosis in order to distinguish chickens infected with influenza viruses from those immunized with inactivated vaccine. These results demonstrate the potential benefit of a simple, specific ELISA for anti-NS1 antibodies that may have diagnostic value for the poultry industries. It is well suited for the large-scale serological surveys. In addition, the simplicity and specificity of the test make the NS1-ELISA a potentially valuable diagnostic tool for AI eradication and a proper candidate in AI controlling programs.

\section{Acknowledgements}

None declared.

\section{Authors' Contribution}

Writer of article: Forough Talazadeh

$\mathrm{PhD}$ thesis of Forough Talazadeh

Directors: Mansoor Mayahi1, Masoudreza Seifi1

Statistical analyser:Mehdi Pourmehdi

\section{Financial Disclosure}

The study was supported by deputy research of Shahid Chamran University of Ahvaz, Ahvaz-Iran.

\section{Funding/Support}

This manuscript was extracted from Phd thesis approved by Shahid Chamran University of Ahvaz. The study was supported by deputy research of Shahid Chamran University of Ahvaz, Ahvaz- Iran.

\section{References}

1. Wright PE, Neumann G, Kawaoka Y, Fields BN, Knipe DM, Howley PM. Orthomyxoviruses. In: Wright PE, Neumann G, Kawaoka Y, Fields BN, Knipe DM, Howley PM, editors.Fields' Virology.Philadelphia, PA: Wolters Kluwer Health/Lippincott Williams \& Wilkins; 2007. p.1692-731.

2. Garcia A, Johnson H, Srivastava DK, Jayawardene DA, Wehr DR, Webster RG. Efficacy of inactivated H5N2 influenza vaccines against lethal A/Chicken/Queretaro/19/95 infection. Avian Dis. 1998;42(2):248-56.

3. Halvorson DA. The control of $\mathrm{H} 5$ or $\mathrm{H} 7$ mildly pathogenic avian influenza: a role for inactivated vaccine. Avian Pathol. 2002;31(1):5-12.

4. Swayne DE, Beck JR, Perdue ML, Beard CW. Efficacy of vaccines in chickens against highly pathogenic Hong Kong H5N1 avian influenza. Avian Dis. 2001;45(2):355-65.

5. Swayne DE. Vaccines for List A poultry diseases: emphasis on avian influenza. Dev Biol (Basel). 2003;114:201-12.

6. Influenza hemagglutination inhibition assay. 2009; Available from: http://www.virology.ws/2009/05/27/influenza-hemagglutination-inhibition-assay/.

7. Suarez DL. Overview of avian influenza DIVA test strategies. Biologicals. 2005;33(4):221-6.

8. Capua I, Terregino C, Cattoli G, Mutinelli F, Rodriguez JF. Devel- opment of a DIVA (Differentiating Infected from Vaccinated Animals) strategy using a vaccine containing a heterologous neuraminidase for the control of avian influenza. Avian Pathol. 2003;32(1):47-55.

9. Capua I, Cattoli G, Marangon S. DIVA--a vaccination strategy enabling the detection of field exposure to avian influenza. Dev Biol (Basel). 2004;119:229-33.

10. Cattoli G, Milani A, Bettini F, Serena Beato M, Mancin M, Terregino $C$, et al. Development and validation of an anti-N3 indirect immunofluorescent antibody test to be used as a companion diagnostic test in the framework of a "DIVA" vaccination strategy for avian influenza infections in poultry. Avian Pathol. 2006;35(2):154-9.

11. Lee CW, Senne DA, Suarez DL. Generation of reassortant influenza vaccines by reverse genetics that allows utilization of a DIVA (Differentiating Infected from Vaccinated Animals) strategy for the control of avian influenza. Vaccine. 2004;22(23-24):3175-81.

12. Tumpey TM, Alvarez R, Swayne DE, Suarez DL. Diagnostic approach for differentiating infected from vaccinated poultry on the basis of antibodies to NS1, the nonstructural protein of influenza A virus. J Clin Microbiol. 2005;43(2):676-83.

13. Capua I, Marangon S. Vaccination policy applied for the control of avian influenza in Italy. Dev Biol (Basel). 2003;114:213-9.

14. Garcia-Sastre A, Egorov A, Matassov D, Brandt S, Levy DE, Durbin JE, et al. Influenza A virus lacking the NS1 gene replicates in interferon-deficient systems. Virology. 1998;252(2):324-30.

15. Suarez DavidL, Perdue MichaelL. Multiple alignment comparison of the non-structural genes of influenza A viruses. Virus Res. 1998;54(1):59-69.

16. Birch-Machin I, Rowan A, Pick J, Mumford J, Binns M. Expression of the nonstructural protein NS1 of equine influenza A virus: detection of anti-NS1 antibody in post infection equine sera. JVirol Methods. 1997;65(2):255-63.

17. Ozaki H, Sugiura T, Sugita S, Imagawa H, Kida H. Detection of antibodies to the nonstructural protein (NS1) of influenza A virus allows distinction between vaccinated and infected horses. Veter Microbiol. 2001;82(2):111-9.

18. Zhao S, Jin M, Li H, Tan Y, Wang G, Zhang R, et al. Detection of antibodies to the nonstructural protein (NS1) of avian influenza viruses allows distinction between vaccinated and infected chickens. Avian Dis. 2005;49(4):488-93.

19. Spackman E. Avian Influenza Virus. Humana Press; 2008. p. 53-65. ISBN 9781588299390.

20. Beard CW, Purchase HG, Arp LH, Domermuth CH, Pearson JE. Serological procedures. In: Beard CW, Purchase HG, Arp LH, Domermuth $\mathrm{CH}$, Pearson JE, editors.A laboratory manual for the isolation and identification of avian pathotypes.Dubuque, Iowa: KendallHunt Publishing; 1989.

21. Hall IH, Burnham BS, Elkins A, Sood A, Powell W, Tomasz J, et al. Boronated pyrimidines and purines as cytotoxic, hypolipidemic and anti-inflammatory agents. Met Based Drugs. 1996;3(3):155-60.

22. Krug RobertM, Etkind PollyR. Cytoplasmic and nuclear virusspecific proteins in influenza virus-infected MDCK cells. Virol. 1973;56(1):334-348.

23. Buonagurio DA, Nakada S, Parvin JD, Krystal M, Palese P, Fitch WM. Evolution of human influenza A viruses over 50 years: rapid, uniform rate of change in NS gene. Science.1986;232(4753):980-2.

24. Ludwig S, Schultz U, Mandler J, Fitch WM, Scholtissek C. Phylogenetic relationship of the nonstructural (NS) genes of influenza A viruses. Virol. 1991;183(2):566-577.

25. Treanor JohnJ, Snyder MarkH, London WilliamT, Murphy BrianR. The B allele of the NS gene of avian influenza viruses, but not the A allele, attenuates a human influenza a virus for squirrel monkeys. Virol.1989;171(1):1-9.

26. Avellaneda G, Mundt E, Lee CW, Jadhao S, Suarez DL. Differentiation of infected and vaccinated animals (DIVA) using the NS1 protein of avian influenza virus. Avian Dis. 2010;54(1 Suppl):278-86.

27. Soleimani R, Vasfi Marandi M, Hashemi-Soteh MB, Hemmatzadeh F. The cloning of non-structural-1(NS1) gene of H9N2 subtype of avian influenza virus in pGEX-4T-1 and pMAL-c2X plasmids and expression in Escherichia coli DH5 $\alpha$ strain. Adv Biosci Biotechnol. 2012;3:283-9. 